\title{
How telemental health delivered to non-traditional locations helped prepare for responses to COVID-19
}

\author{
Matthew C. Mishkind \\ Departments of Psychiatry and Family Medicine, Helen and Arthur E Johnson Depression Center, School of Medicine, University of Colorado \\ Anschutz Medical Campus, Aurora, CO, USA \\ Correspondence to: Matthew C. Mishkind, PhD. Departments of Psychiatry and Family Medicine, Helen and Arthur E Johnson Depression Center, \\ School of Medicine, University of Colorado Anschutz Medical Campus, Aurora, CO, USA. Email: matthew.mishkind@cuanschutz.edu.
}

Received: 27 March 2020; Accepted: 15 May 2020; Published: 20 April 2021.

doi: $10.21037 /$ mhealth-2020-4

View this article at: http://dx.doi.org/10.21037/mhealth-2020-4

\section{Introduction}

The COVID-19 pandemic has triggered significant changes across societies and healthcare systems worldwide through a range of isolation, suppression, and mitigation strategies (1). Disasters can exacerbate already known mental health concerns and the continued provision of mental health services is important during public health emergencies (2). A recent review evaluating the impact of quarantine/ self-isolation, similar to measures used to suppress and mitigate COVID-19 spread, found that most studies reported negative psychological effects including posttraumatic stress symptoms, confusion, anger, and other concerns associated with frustration, boredom, fear, and loss of financial and other resources (3). Telemental health (TMH), defined here as the use of technology to provide mental health care at a geographic or temporal distance, has fostered the mental health care community's ability to adapt quickly to these mitigation strategies and has allowed for the continued provision of mental health services during this pandemic.

TMH has a broad evidence base for clinical efficacy and has been shown to make significant positive impacts on the efficiency of mental health delivery systems by more effectively tailoring mental health services to individual or community-based health care needs (4-7). This includes the promotion of a stepped care approach that provides beneficiaries with a range of specialized services such as mobile applications, web-based, and other computerassisted services for some issues (8), along with direct person-to-person connections with a mental health provider for more complex and specialized concerns. The flexibility to tailor services to individual and community access needs made TMH uniquely positioned for success during this global crisis.

\section{Traditional and non-traditional settings}

Early TMH systems used what were often costly and, at times, unreliable synchronous two-way, audio and video connections, and the infrastructure of these early programs required the resources of large institutions to be effective (9). Therefore, TMH systems were traditionally based on providing mental health care from one clinically supported institutional setting (e.g., hospital or clinic) to another. Fortunately, TMH has evolved rapidly over the past two decades concurrently with revolutions in devices such as smartphones and communications technologies including the Internet, and overall changes in the healthcare landscape focused on improving care and access while reducing costs. The acceptability of service locations has also broadened beyond institutional walls as many of the technologies and peripherals used to deliver services today are ubiquitous to consumers based largely on commercial use and applications (8).

There is no absolute definition for what may be considered a "non-traditional setting" for TMH services, although it may be argued that a service location different from the traditional hospital or clinic setting norm qualifies. Another definition may suggest that care delivered to clinically unsupported settings such as patient homes may also be non-traditional as many systems, insurers, and even providers have been reluctant to deliver services to these 
clinically unsupported settings. Responses to COVID-19 indicate that the definition of traditional and non-traditional locations is fluid and can evolve rapidly especially when there is a defined need for change.

\section{In-bome TMH}

Although providing TMH services direct to a patient's home, as part of a systematic program, is a more recent development there already exists good evidence of its efficacy across mental health concerns, patient populations, and ages (10-15). Much like the development of clinic-toclinic TMH, the evolution of home-based care began with pilot programs that provided use cases and best practices that led to industry guidelines, programmatic development, and insurance reimbursements. While it may be argued that few differences exist clinically between clinic-toclinic and home-based services, there have been questions about the overall feasibility of in-home TMH as defined by treatment adherence, patient and provider satisfaction, cost effectiveness, and other clinical considerations. The current evidence available suggests that in-home TMH is not only clinically effective but also a feasible alternative to both inperson and more traditional clinic-based TMH (10).

\section{Applying lessons learned to COVID-19 responses}

Many mental health organizations and providers utilized $\mathrm{TMH}$ to transition from in-person to virtual services to support COVID-19 suppression and mitigation strategies. The existing evidence suggests that clinically unsupported settings, particularly patient homes, are effective sites of care (10-15) was instrumental to this transition. The lifting of many regulatory barriers to telehealth, even temporarily along with provider flexibility and patient demand, further facilitated this rapid virtual transformation. Although TMH has been previously leveraged temporarily in disaster response (16), its use during the COVID-19 pandemic is distinctive by its widespread deployment and presumably longer-lasting impact in how providers and systems deliver services. While the change to fully virtualized care may be temporary for some, the range of systems now promoting TMH solutions in varied settings makes it unlikely that we will return to strict boundaries for what defines the setting of care. Several resources are available that provide guidance for developing TMH services and establishing appropriate guidelines $(17,18)$. Some lessons learned from in-home $\mathrm{TMH}$ research and the provision of care to clinically unsupported settings and in response to COVID-19 are provided below.

\section{Safety considerations}

There may be an argument that providers have limited control over clinically unsupported settings and encounters compared to traditional in-person sessions. However, this argument does not represent the real amount of control a provider or other clinical and administrative staff have both in session and during the significant time periods between sessions when there is limited or no contact with a patient. Furthermore, for some patients a TMH encounter may be the only direct connection available with a provider, as is the case for many during the current period of COVID-19 isolation.

TMH session standards occur within the context of the environment mutually agreed upon between patient and clinician. This includes locations with well-established safety protocols, as well as those requiring additional competence to manage such as clinically unsupported settings. There is direct evidence that safety concerns are not only managed by use of established procedures but may also provide the necessary link to someone in crisis. Gros and colleagues (19) provided one of the first reports of use of in-home TMH services to identify suicidality in a patient and intervene with a safety plan using a series of enhanced communications. The authors suggest that the telehealth equipment provided three benefits during the emergency: (I) a secondary route of communication for the provider, allowing for external safety planning to occur simultaneously; (II) constant visual observation for ongoing assessment of distress; and (III) observation of other potentially self-harming behaviors. The use of in-home TMH in this situation was instrumental to, rather than inhibiting of patient safety management, and demonstrated that care delivered to clinically unsupported settings can be safely managed with appropriate plans and protocols.

\section{Identifying needs}

Guidelines suggest that a needs assessment should be conducted prior to initiating TMH services as programs tend to fail when planners do not properly understand the problem that $\mathrm{TMH}$ is attempting to solve $(17,18)$. The use of non-traditional settings in situations such as the response to COVID-19 may indicate the need to develop resources 
quickly and may not provide time for the implementation of a full assessment. Whether a program is being developed quickly for shorter-term needs (e.g., COVID-19) or for more systematic programs (e.g., in-home TMH 6-months post COVID-19), first understand the intent of the service and what you want to offer. Working through what is likely to be a typical clinical visit, from before the encounter to referral and follow-up, is a recommended pathway in the process. Next, evaluate the following at a minimum.

* Personnel resources: determine if you have providers and others on staff who are able and willing to deliver TMH care services to nontraditional locations.

* Technology needs: the services to be provided should drive the technology, rather than the opposite.

* Operational space: where the patient is located (the originating site) rather than where the provider is located (the distant site) is where care is delivered and plans for providing services in one jurisdiction will not immediately equate to a similar plan in another location.

* Regulations: it is critically important to understand the legal and regulatory requirements for your originating and distant site operational spaces including licensure, credentialing and privileging, malpractice insurance, and HIPAA and other related regulations.

* Leadership support: engaged leaders that are supportive of virtual services are critical to ongoing success.

\section{Training and education}

Provider, and other staff, and potentially patient training and education should be a focal point when implementing any new service. This is especially true in clinically unsupported settings where operating procedures and environments may differ from standard practice and there may be more limited synchronous communications virtually. Limiting training to providers may hinder the development of a new service program, and it is recommended to evaluate the training needs of all staff. One study evaluated a training protocol for a deploying United States Army unit tasked to expand TMH services in Afghanistan and suggested five training best practices before working in a non-traditional setting (20). The overarching theme is that interactive training in real-life scenarios and implementing real-time standard operating processes an invaluable. The practices, updated for more current implementation needs, are reviewed below.

(I) Use already established didactic training materials for topics such as TMH background, history, and evidence-base.

(II) Focus on live, interactive sessions for the practice of trouble-shooting technology issues, practicing rapport-building techniques, and implementing standard operating procedures in the new environment.

(III) Use lessons learned from other known programs or research to develop real-to-life training scenarios that represent the non-traditional location and needs.

(IV) Incorporate, if possible, training into daily activities such as holding meetings via videoconferencing rather than limiting to a training session.

(V) Tailor training based on roles while ensuring that all staff have the same base competencies and knowledge sets.

\section{Other considerations}

Operating in non-traditional locations, especially those that are clinically unsupported such as patient homes, requires collaboration between clinical and administrative teams. This can be done through use of variety of communication technologies to ensure continuity of operations. Employing staff and providers already experienced in TMH and managing a virtual workforce will further benefit these collective efforts. The willingness to engage in and capacity for rapid learning is vitally important to foster communication and connection between staff. Potentially most important is to monitor employee morale as some work in non-traditional locations may be isolating or otherwise taxing. Finally, focus on immediate tasks that make sense without overcomplicating solutions. This can help translate to a thoughtful, strategic, and measured approach. For example, working from already established protocols and modifying only what is necessary to engage in TMH services while leaving many aspects of standard operating procedures unchanged will support the transition to virtual services.

\section{Concluding thoughts}

Mental health systems world-wide will be transformed when 
we emerge from the current pandemic and we expect much wider use and acceptance of TMH into the foreseeable future. There will always be a need for efficacy research when delivering services to new locations. However, the concept of "non-traditional" is likely to change and what was once new will be the new normal. Social distancing, isolation, and the deployment of suppression and mitigation strategies will not just directly influence the future morbidity and mortality of the pandemic but will also force health care providers and systems to re-conceptualize how, when, and where services are provided. For example, in-home TMH is rapidly gaining acceptance along with providers delivering services from their own homes. It is not too difficult to imagine how the information gained today will lead to acceptance of TMH originating sites such as individual offices and hotels in the not too distant tomorrow. It will be important for us to use the lessons learned now to continue to improve access and quality in ways that benefit patients, colleagues, and community members in the future. It is also important to understand the different patient and provider perspectives and other virtualized service solutions to foster a future where settings are fluid and services delivered beyond traditional walls are the norm.

\section{Acknowledgments}

Funding: None.

\section{Footnote}

Provenance and Peer Review: This article was commissioned by the editorial office, $m$ Health for the series "Developing telehealth programs for remote and isolated populations". The article did not undergo external peer review.

Conflicts of Interest: The author has completed the ICMJE uniform disclosure form (available at http://dx.doi. org/10.21037/10.21037/mhealth-2020-4). The series "Developing telehealth programs for remote and isolated populations" was commissioned by the editorial office without any funding or sponsorship. MCM served as the unpaid Guest Editor of the series. MCM reports serving as a paid advisory board member for Meta Pro. This relationship is managed through the University of Colorado School of Medicine. The author has no other conflicts of interest to declare.
Ethical Statement: The author is accountable for all aspects of the work in ensuring that questions related to the accuracy or integrity of any part of the work are appropriately investigated and resolved.

Open Access Statement: This is an Open Access article distributed in accordance with the Creative Commons Attribution-NonCommercial-NoDerivs 4.0 International License (CC BY-NC-ND 4.0), which permits the noncommercial replication and distribution of the article with the strict proviso that no changes or edits are made and the original work is properly cited (including links to both the formal publication through the relevant DOI and the license). See: https://creativecommons.org/licenses/by-nc-nd/4.0/.

\section{References}

1. Anderson RM, Heesterbeek H, Klinkenberg D, et al. How will country-based mitigation measures influence the course of the COVID-19 epidemic? Lancet 2020;395:931-4.

2. Mollica RF, Cardozo BL, Osofsky HJ, et al. Mental health in complex emergencies. Lancet 2004;364:2058-67.

3. Brooks SK, Webster RK, Smith LE, et al. The psychological impact of quarantine and how to reduce it: rapid review of the evidence. Lancet 2020;395:912-20.

4. Bashshur RL, Shannon GW, Bashshur N, et al. The Empirical Evidence for Telemedicine Interventions in Mental Disorders. Telemed J E Health 2016;22:87-113.

5. Hilty DM, Ferrer DC, Parish MB, et al. The effectiveness of telemental health: a 2013 review. Telemed J E Health 2013;19:444-54.

6. Hubley S, Lynch SB, Schneck C, et al. Review of key telepsychiatry outcomes. World J Psychiatry 2016;6:269-82.

7. Mishkind M, Waugh M, Hubley S. Evidence base for use of videoconferencing and other technologies in mental health care. In: Yellowlees P, Shore JH. editors. Telepsychiatry and Health Technologies: A Guide for Mental Health Professionals. 2018.

8. Wright JH, Mishkind M, Eells TD, et al. ComputerAssisted Cognitive-Behavior Therapy and Mobile Apps for Depression and Anxiety. Curr Psychiatry Rep 2019;21:62.

9. Shore J. The evolution and history of telepsychiatry and its impact on psychiatric care: Current implications for psychiatrists and psychiatric organizations. Int Rev Psychiatry 2015;27:469-75.

10. Fletcher TL, Hogan JB, Keegan F, et al. Recent Advances 
in Delivering Mental Health Treatment via Video to Home. Curr Psychiatry Rep 2018;20:56.

11. Comer JS, Furr JM, Miguel EM, et al. Remotely delivering real-time parent training to the home: An initial randomized trial of Internet-delivered parent-child interaction therapy (I-PCIT). J Consult Clin Psychol 2017;85:909-917.

12. Morland LA, Mackintosh MA, Glassman LH, et al. Homebased delivery of variable length prolonged exposure therapy: A comparison of clinical efficacy between service modalities. Depress Anxiety 2020;37:346-55.

13. Carpenter AL, Pincus DB, Furr JM, et al. Working From Home: An Initial Pilot Examination of Videoconferencing-Based Cognitive Behavioral Therapy for Anxious Youth Delivered to the Home Setting. Behav Ther 2018;49:917-30.

14. Boykin DM, Keegan F, Thompson KE, et al. Video to Home Delivery of Evidence-Based Psychotherapy to Veterans With Posttraumatic Stress Disorder. Front Psychiatry 2019;10:893.

doi: 10.21037/mhealth-2020-4

Cite this article as: Mishkind MC. How telemental health delivered to non-traditional locations helped prepare for responses to COVID-19. mHealth 2021;7:17.
15. Whealin JM, King L, Shore P, et al. Diverse veterans' preand post-intervention perceptions of home telemental health for posttraumatic stress disorder delivered via tablet. Int J Psychiatry Med 2017;52:3-20.

16. Augusterfer EF, Mollica RF, Lavelle J. A review of telemental health in international and post-disaster settings. Int Rev Psychiatry 2015;27:540-6.

17. Shore JH, Yellowlees P, Caudill R, et al. Best Practices in Videoconferencing-Based Telemental Health April 2018. Telemed J E Health 2018;24:827-32.

18. Mishkind MC. Establishing Telemental Heath Services from Conceptualization to Powering up. Psychiatr Clin North Am 2019;42:545-54.

19. Gros DF, Veronee K, Strachan M, et al. Managing suicidality in home-based telehealth. J Telemed Telecare 2011;17:332-5.

20. Mishkind MC, Boyd A, Kramer GM, et al. Evaluating the benefits of a live, simulation-based telebehavioral health training for a deploying army reserve unit. Mil Med 2013;178:1322-7. 